\title{
BIOÉTICA Y MUJER: UN ANÁLISIS IUSFILOSÓFICO DE LA DIGNIDAD HUMANA COMO CONDICIÓN DE EQUIDAD
}

\author{
BIOETHICS AND WOMEN: \\ A IUSPHILOSOPHICAL ANALYSIS OF HUMAN DIGNITY AS EQUITY \\ CONDITION
}

\author{
Diana Milena Gómez Hurtado \\ Corporación Universitaria Remington \\ ORCID: 0000-0001-9807-6318 \\ diana.gomez@uniremington.edu.co \\ Colombia
}

\section{SUMARIO}

- Introducción

- Bioética y dignidad de la mujer

- La dignidad de la mujer y la apuesta por la equidad

- La dignidad humana de la mujer como una apuesta inclusiva por la vida: análisis sobre el aborto

- Conclusiones

- Fuentes de Información

\section{RESUMEN}

La Bioética se relaciona con distintas prácticas y modos de vida en las que se presenta un pluralismo ético, pero a pesar de la diversidad y el relativismo que esto presenta, el tratamiento y fundamento que se dé a la vida debe tener como límite el reconocimiento de la dignidad humana dado que este principio funciona como garante del respeto por los seres humanos y garantiza la supervivencia en condiciones de autonomía y libertad. Dentro de los escenarios en los que se hace necesario reconocer la dignidad humana, se encuentra la consideración de la vida y los derechos de las mujeres, quienes necesitan una sociedad en la que su existencia sea siempre vista como un fin y no como un medio. El análisis filosófico sobre las mujeres y la bioética supone comprender que "ellas" no son un instrumento y que, como tal, sus decisiones, pensamientos y luchas son un modo de construir vida que requiere, al igual que en otros géneros $y$ grupos sociales, de oportunidades y garantías que les permitan desarrollarse y avanzar en la sociedad en condiciones de equidad. Mediante el método hermenéutico se realiza una interpretación de los retos que impone la bioética a la mujer para construir su identidad y defender su dignidad.

\section{ABSTRACT}

Bioethics is related to different practices and lifestyles in which ethical pluralism is presented, but despite the diversity and relativism that it presents, the treatment and basis that we give to the life must be limited by the recognition of human dignity, since this principle serves as a guarantor of respect for human beings and ensures survival under conditions of autonomy and freedom. Within the scenarios where it is necessary to recognize human dignity, we found the respect for the life and women 
Bioethics and women: A iusphilosophical analysis of human dignity as equity condition.

rights, who need a society in which their existence be always seen as an end and not as a means. Philosophical analysis of women and bioethics implies to understand that "they" are not an instrument, besides their decisions, thoughts and struggles are a way of building life that requires, as in other genders and social groups, opportunities and guarantees that allow them to grow up and develop in society in conditions of equity. The hermeneutic method provides an interpretation of the challenges that bioethics imposes on women to build their identity and defend their dignity.

\section{PALABRAS CLAVE}

Bioética; dignidad humana; equidad; mujer.

\section{KEYWORDS}

Bioethics; equity; human dignity; woman.

\section{INTRODUCCIÓN}

El sujeto se construye a sí mismo, a los otros y a lo otro, bajo la influencia de una sociedad en la que convergen supuestos éticos que legitiman o no sus acciones. Si ese sujeto es una mujer, su construcción de sí misma es un ejercicio bioético influenciado por las singularidades de su comunidad, su disposición biológica y su lucha por ser receptora de derechos que le impliquen una participación equitativa en la sociedad. El sujeto mujer que se construye con dependencia a las circunstancias sociales, familiares y temporales elabora una sinergia entre su yoeidad, la realidad y el papel que debe desempeñar como actora y receptora de sus circunstancias, lo que le implica reflexionar constantemente sobre ser mujer y construirse mujer.

En el ejercicio bioético que establece la mujer sobre sí misma y la forma como es reconocida socialmente, el principio de dignidad humana representa su principal lucha como actora y receptora de derechos con pleno ejercicio de su autonomía y libertad. Por ello, un derecho como la salud sexual y reproductiva implica la defensa de la dignidad humana porque permite la práctica de la libertad de decisión sobre la sexualidad y la maternidad. Si bien es relevante la lucha por estos derechos, la defensa de la mujer en condiciones de dignidad y equidad exige también pensarle dentro de una sociedad en la que se encuentran presentes los mismos derechos que ella reclama y son igualmente exigibles para otros sujetos que también se encuentran en circunstancias de vulnerabilidad. Por lo anterior, el presente texto busca realizar una crítica iusfilosofica al aborto, en tanto se convierte en una práctica de dignidad humana que elimina la dignidad humana, es decir, en una defensa de derechos y libertades que eliminan la posibilidad misma de ejercerlos aniquilando la vida.

\section{BIOÉTICA Y DIGNIDAD DE LA MUJER}

\section{Oh! de un rostro a otro rostro cuánta elevación. \\ Irrumpe en los culpables remisión y renuncia.}

(Rilke,1956, p.27)

La bioética como principio investigativo implica otorgar un tratamiento digno al ser humano que es objeto de investigación y, con ello, ahondar en el individuo desde sus particularidades y diferencias, desde la manera como se construye éticamente y se perfila corporal y socialmente. La bioética permite asumir una perspectiva de inclusión y reconocimiento de diferencias humanas $\mathrm{y}$, de esta forma, construir una mirada crítica desde la cual estudiar la condición humana, como es el caso de ser mujer, en tanto que la vida de ellas dentro de la especie humana es la más expuesta a las transformaciones y a las criticas éticas, en gran medida porque es la mujer la que más se enfrenta a cambios estéticos, como 
ocurre con las elecciones y disposiciones frente a la maternidad. Lo anterior se explica en que históricamente, según Delgado Díaz (2008), la bioética refiere a un principio en investigación que parte de los:

Problemas generados no sólo en las ciencias de la salud por la implementación de las nuevas tecnologías sobre la salud humana, sino en todas las ciencias implicadas en el desarrollo de la vida en todas sus manifestaciones. Se proponía con ella el establecimiento de una ética de la vida, que, partiendo de unos principios generales, tomara en cuenta y facilitara soluciones a problemas de orden bioético, otorgándole el derecho a toda persona implicada a decidir $\mathrm{y}$ participar en la resolución de los mismos. (p.1)

La bioética ubica a la mujer frente a las nuevas tecnologías que le implican repensar su rol frente a la sociedad y a su vez reflexionar sobre cuál es su papel en el desarrollo de la vida. Por tanto, siempre la bioética representa una manera en la que la mujer se asume como parte de la creación y la modificación de la vida, lo que implica que un tema como la maternidad es transversalmente bioético en tanto establece una pluralidad moral que se fundamenta en la diversidad de pensamientos que surgen bajo distintos contextos de vida.

La bioética se ha preocupado desde sus inicios por "los derechos del enfermo, sobre todo en su condición de persona vulnerable que no por ello pierde su integralidad, individualidad y autonomía"(Boscán Leal, 2015,p.166) lo que significa defender la vida humana frente a las condiciones de debilidad, preocupación que ha sido evidente desde el origen de la bioética, por lo que si se habla de la mujer como un sujeto vulnerable, se identifica la necesidad de reconocerle y posicionarle socialmente y ello solo es posible a través de la inclusión de derechos y oportunidades sociales que le permitan a la mujer ejercer su autonomía y libertad y, de esa manera, construirse como un sujeto ético.

Además de la reflexión sobre la vida humana desde los derechos y experiencias, la bioética también guarda relación con los problemas morales que implican una mezcla transdisciplinar entre la explicación científica y la reflexión filosófica, lo que permite hacer de la bioética una disciplina que posibilita "alcanzar una consciencia integral de nuestra experiencia como seres humanos, basándose en la dignidad de la persona y en el valor de la vida para la existencia plena de un ser inserto en una cultura y con creencias particulares”(Boscán Leal, 2015,p.167). Se trata entonces de un acercamiento abierto a todo problema sobre la vida que requiere de investigación y debate $\mathrm{y}$, en esa medida, resulta plausible comprender que la condición de vivir siendo mujer tiene sentido filosófico, dado que la vivencia de lo femenino implica comprender a la dignidad humana y a la libertad como principios y como fines de toda vida, asi como fundamentos de la construcción de equidad en una sociedad.

La necesidad de reconocimiento equitativo de la mujer hace necesario considerar cuáles son los aspectos particulares de la vida de las mujeres y sus exigencias éticas. Al respecto se pregunta Figueroa (2017) si:

¿existe una ética idéntica que prescriba las reglas que regulen las relaciones con la mujer y con el varón? Por su parte, durante las últimas décadas la bioética se ha ido adentrando progresivamente en este problema de manera empírica, empujada por apremios clínicos, presiones sociales $\mathrm{y}$ exigencias culturales. Empero, al intentar 
Bioethics and women: A iusphilosophical analysis of human dignity as equity condition.

resolverlo, lo ha llevado a cabo sin cuestionarse por los fundamentos antropológicos que están en la base de las diversas perspectivas, propuestas y respuestas( p.84)

La manera como la sociedad trata tanto a los hombres como a las mujeres no tiene un perfil de vida ético de iguales proporciones, cada uno posee unas exigencias sociales y culturales que condicionan y legitiman su accionar y que, en múltiples ocasiones, también posibilitan generar espacios de inequidad. Así, por ejemplo, las exigencias sobre el cuerpo, la limpieza, la delicadeza, el trato, son socialmente distintas, legitimando así tratos inequitativos. Es por eso que, al pensar bioéticamente el ser mujer, se requiere del análisis sobre qué es la dignidad humana y, concretamente, sobre qué es lo que hace a la mujer digna y cuáles son los derechos y los comportamientos que permiten garantizar dicha dignidad. Solo desde esta hermenéutica social y filosófica, puede comprenderse por qué al reconocer la dignidad del ser mujer se desvirtúa la inequidad social que frente a ella se practica.

Primeramente, es necesario precisar a qué hace referencia el concepto de dignidad humana, dado que se ha creído que "en nuestro margen cultural es hegemónica la representación de la dignidad humana como un concepto de contenido inequívoco y además, universal." (Londoño, 2012, p.18) lo que hace necesaria su delimitación conceptual. Desde el filósofo alemán Immanuel Kant, la dignidad refiere al trato para con los seres humanos, el cual debe permitirles su desarrollo como personas. La dignidad se entiende partiendo de la consideración de la libertad como atributo humano de otorgarse leyes morales a partir de un ejercicio libre y autónomo para el direccionamiento de sus acciones. No se trata de un atributo exclusivo y excluyente de una determinada forma de entender el mundo, sino que ha de valer para todo ser racional en general y, por tanto, lo que está implícito es una moral antropológica cuyo imperativo es el reconocimiento del ser humano y su capacidad para autodeterminarse

La autonomía que subyace a la dignidad humana consiste en darse una ley a sí mismo, en ser capaz de actuar pensando en lo que otros harían en similares circunstancias. Refiere además a la comprensión de que para acercarnos a la forma como otros actúan y dirigen sus acciones, vale simplemente el ejercicio de la razón; pero también supone entender que "si lo consideramos todo desde uno y el mismo punto de vista, a saber, el de la razón, hallaremos una contradicción en nuestra propia voluntad, a saber: que cierto principio es necesario objetivamente como ley universal, $\mathrm{y}$, sin embargo, no vale subjetivamente con universalidad, sino que ha de admitir excepciones." (Kant,2007, p. 38), y esas excepciones son, precisamente, las elecciones vitales y las prácticas concretas de razonabilidad, las cuales aunque pareciesen repudiables, no son más que la libre expresión de la intimidad de un sujeto que se asume con particularidad.

Así ocurre con las mujeres, quienes tienen capacidad para elegir con autonomía y desarrollar diversas prácticas sobre sí mismas, como ocurre con la libertad de desarrollo de la personalidad, la libertad de elegir culto y las libertades sexuales y reproductivas. La idea clásica de la moral kantiana que expresa: "obra según la máxima que pueda hacerse a sí misma al propio tiempo una ley universal"(Kant, 2007 p.50) ha sido el fundamento para convertir el concepto de dignidad humana en una idea absolutista del poder, de la sumisión y de la globalización, pero como ya se precisó, no todo sujeto puede inmiscuirse en un supuesto ideario de universalidad, porque cada uno según su propia visión del mundo determina lo que ha de ser bueno o malo según su propia microuniversalidad. 
Esta realidad ha llevado a que los mismos derechos humanos no sean aplicables de igual manera en hombres y mujeres y que deban existir normas internacionales que precisen el alcance de los derechos humanos en las mujeres, como lo es la Convención Interamericana para Prevenir, sancionar y erradicar la violencia contra la mujer "Convención de Belem do Pará" (1994), en la que se estipula la obligatoriedad de los Estados para frenar y sancionar la discriminación y las distintas formas de violencia contra la mujer, prevaleciendo con ello el reconocimiento de la dignidad humana de la mujer desde el escenario jurídico y político.

\section{LA DIGNIDAD DE LA MUJER Y LA APUESTA POR LA EQUIDAD}

La dignidad humana exige considerar a la mujer como un sujeto libre, racional, autónomo, que se somete a su propia legislación, es decir a su autonomía; porque ella es un fin en sí mismo y no un medio y es por ese valor que toda mujer merece respeto moral y no ser sometida a acciones de discriminación. El reconocimiento de la dignidad en las mujeres es importante porque representa la aceptación de su capacidad para decidir sobre sí misma, para actuar con autonomía y para defender y ser receptora de sus múltiples derechos. Así, por ejemplo, la Convención de Belém do Pará, identifica que el respeto por la dignidad de la mujer inicia por el respeto a sus derechos humanos y, por tanto, por la eliminación de toda forma de violencia hacia ella. La vulneración de la dignidad de la mujer perpetúa "las relaciones de poder históricamente desiguales entre mujeres y hombres...trasciende todos los sectores de la sociedad independientemente de su clase, raza o grupo étnico, nivel de ingresos, cultura, nivel educacional, edad o religión y afecta negativamente sus propias bases"( Convención Interamericana para Prevenir, Sancionar y Erradicar la Violencia contra la Mujer, 1994)
El respeto por los derechos y la dignidad de la mujer, le permite hacer parte activa de la construcción de sociedad y participar libremente de los distintos escenarios de configuración política. La discriminación, la violencia y la subvaloración se han hecho costumbre en la sociedad, precisamente por la ausencia de valores y de valoración del papel protagónico de la mujer en la sociedad, lo cual hace necesario repensar la inclusión como una manera de fortalecer los valores humanos y comunitarios. $\mathrm{La}$ dignidad que se le otorga a la mujer al reconocerle el pleno ejercicio de sus derechos corresponde con una apuesta por el fortalecimiento de los valores y los derechos humanos.

La no discriminación de la mujer como apuesta por la efectividad de los derechos humanos, corresponde con la sustentabilidad social, es decir, con la determinación de que las mujeres poseen las capacidades y potestades jurídicas de realizar actividades igual de productivas que los hombres y para ser portadoras de un mensaje social de respeto, tolerancia, igualdad y equidad. La sociedad se sostiene en el tiempo con la aceptación de un orden político el cual, de ser discriminatorio, solo puede desembocar en luchas, desequilibrios sociales y empoderamientos de género.

La dignificación de la mujer como prioridad política con exigencia social y jurídica, debe iniciar por la lucha contra los imaginarios sociales que perpetúan la discriminación, en especial aquellos que configuran una idea de mujer destinada al goce y ubicada al margen de la producción y el conocimiento. Así, por ejemplo, existen escenarios de la publicidad en los cuales se hace uso de la imagen femenina como prototipo comercial y se hace del cuerpo de la mujer un instrumento de cosificación de su belleza, del ser mujer se hacen medidas y se estigmatizan comportamientos, se visiona a la mujer como un medio de bienestar, estabilidad, fantasía, pero se desconoce los fines de producción, 
Bioethics and women: A iusphilosophical analysis of human dignity as equity condition.

creación, innovación y conocimiento, que subyacen en las actividades cotidianas que desarrollan. Lo anterior configura una idea de mujer anclada a permitir la exclusión de sus derechos $y$ a permitir que sean reemplazos por hechos de necesidad comercial. Dignificarse supone asumirse como un sujeto de derechos, capaz de actuarlos y promoverlos.

Al tratarse de un ser digno, la mujer tiene un valor absoluto y, como tal, debe ser equitativo respecto a los demás géneros humanos. "La mujer es sujeto de derechos y esto implica la exigencia de protección coercitiva a través de la normatividad jurídica" (Casado, M, Luna, F y Vásquez, R, 2013, p.19). Desde la bioética reconocer los derechos implica a su vez reconocer unas prácticas de vida que les son propias a las mujeres como lo son sus distintas construcciones éticas.

La dignidad humana de la mujer implica el respeto por su cuerpo y su privacidad, ello se relaciona con la importancia de reconocer sus derechos a las libertades sexuales y las libertades reproductivas. Al respecto, el ejemplo más emblemático en la Corte Constitucional de Colombia se encuentra, en palabras de Casado, M, Luna, F y Vásquez (2013) en:

Un fallo del 10 de mayo de 2006. En este fallo se destacó la no equivalencia entre el nasciturus y la vida humana de la mujer con todos los derechos de esta última: a su cuerpo, a su sexualidad y reproducción, a su intimidad, etcétera. p. 21.

Lo que se encuentra en el seno de la discusión frente a las libertades sexuales y las libertades reproductivas es su libre desarrollo, que como persona adquieren las mujeres, ese que para la Corte Constitucional en la Sentencia T-222 de 1992 refiere a "libertad general de actuar" (Corte Constitucional, Sala Plena,
Sentencia T-222 de 1992). Otras Sentencias han sostenido de forma similar, las siguientes definiciones: "Libertad general para actuar o no actuar" (Sentencia T-493 de 1993), "libertad in nuce.. porque cualquier libertad se reduce finalmente a ella" (Sentencia C-221 de 1994. M.P Carlos Gaviria Díaz) y como "cláusula general de libertad" (Sentencia T-090 de 1996. MP. Eduardo Cifuentes). En todas ellas se evidencia su compleja naturaleza y su indeterminado espacio de aplicación y por ello es necesario precisar si este principio es absoluto y se corresponde por completo con la dignidad humana.

\section{LA DIGNIDAD HUMANA DE LA MUJER COMO UNA APUESTA INCLUSIVA POR LA VIDA: ANÁLISIS SOBRE EL ABORTO}

La falta de determinación y limitación del derecho a la libertad en la formación de la personalidad ha impedido la efectividad de la defensa de los derechos reproductivos de la mujer, los cuales "tienen su ancla conceptual a nivel internacional en la Conferencia Internacional realizada en 1994 sobre la Población y el Desarrollo (CIPD). Programa de Acción que podría decirse tiene la primicia al reclamar la probidad legal internacional sobre el término "derechos sexuales" (Miller y Roseman, 2011).

Los derechos sexuales y reproductivos se ven especialmente vulnerados en las mujeres. Así por ejemplo, los escenarios de violencia sexual evidencian las condiciones de inequidad frente a este derecho. Las agresiones sexuales contra las mujeres son una problemática que ha comenzado a ser notable públicamente desde los años 70 con las luchas feministas interesadas en visibilizar las distintas situaciones $y$ expresiones de violencia a las que las mujeres eran sometidas tanto en el hogar como en otros escenarios sociales, demostrándose una realidad que logró sobrepasar el espacio privado de las 
mujeres e ingresar al escenario público de los organismos internacionales. Desde entonces, la problemática ha sido objeto de estudio a tal punto que "la proliferación de estudios y programas dirigidos a enfrentar esta problemática impulsó cambios tanto en la manera de tratar la violencia familiar y sexual como en la percepción de las personas" (Fuller, 2008).

La denigración a la que se ve expuesta la mujer frente a los actos de violencia sexual, resulta una violación concreta a la dignidad humana, toda vez que, como afirma Gil (2015):

La concepción de dignidad humana, constituye la gestación del principio de igualdad, inherente a todas las personas sin distinción de raza y sexo, que comporta el deber indeclinable de otorgarle a mujeres y hombres los mismos derechos, por lo que desde esta perspectiva es inconcebible la necesidad de generarse otros derechos o acciones positivas a favor de uno u otro género, sin embargo, esta realidad no aplica para el género femenino, como consecuencia de una histórica subvaloración.p. 817

La condición de inferioridad que se ha otorgado a la mujer es el resultado de una cultura que privilegia el androcentrismo y ubica al hombre como el centro de todo, mediante "perspectivas parciales, androcéntricas, que se imponen [y que] no han considerado ni los puntos de vista ni las experiencias de las mujeres, lo que ha resultado en la invisibilización de las violaciones cotidianas a sus derechos humanos" (Gil, 2015, p. 817). Todo ello da paso a la aceptación de la discriminación contra la mujer y su reduccionismo.

La especial vulnerabilidad de la mujer a la discriminación ha sido evidente para la jurisprudencia de la Corte Interamericana de Derechos Humanos, Así, por ejemplo, la
Corte reconoce que "la libertad y autonomía de las mujeres en materia de salud sexual y reproductiva ha sido históricamente limitada, restringida o anulada con base en estereotipos de género negativos $y$ perjudiciales" (Sentencia C No. 329 de 2016, Corte IDH. Caso I.V. Vs. Bolivia) lo cual se hace especialmente evidente en el rol privilegiado que ubica a los hombres en la toma de decisiones sobre el cuerpo y las relaciones sociales de las mujeres.

La desigualdad para con las mujeres se evidencia también en la sexualidad, al punto de llegar a cosificarla y, con ello, justificar los sometimientos, insinuaciones y contactos de naturaleza sexual no consentidos por las mujeres. Las actuaciones que configuran las distintas formas de violencia sexual atentan contra los bienes jurídicos como son la libertad sexual y la libertad reproductiva, los cuales se encuentran amparadas en el derecho internacional y nacional. Lo alarmante es que la violencia contra la mujer "no se limita a la violación de sus derechos o libertades, pues, la magnitud del daño trasciende la facultad que tiene la mujer de decidir responsable, voluntaria y libremente la forma de ejercer su sexualidad" (Gil, 2015, p. 818) aún con lo anterior, estas acciones se enmarcan dentro de la antijuricidad y la magnitud del daño, por lo que implican una responsabilidad jurídica y social.

La violencia sexual que se efectúa contra las mujeres y sus libertades sexuales y reproductivas, ponen sobre la balanza un tema como el aborto, dado que en el escenario de un abuso sexual, el aborto es un derecho y no un delito. Pero el debate sobre el aborto va más allá de la libertad y la violencia, dado que es un asunto que toca con la responsabilidad social, la educación, el plano ético y religioso, lo que hace más complejas las modificaciones y precisiones legislativas que sobre este tema desarrollan los Estados 
Bioethics and women: A iusphilosophical analysis of human dignity as equity condition.

Lo relevante aquí es que, más allá de los prejuicios y los confinamientos sociales, el aborto toca con la dignidad humana y con la configuración de su identidad y libertad sexual. Por tanto, debe medirse de manera preeminente desde el libre ejercicio y formación de la personalidad, entendiendo que lo que está en juego en un tema como el aborto es la capacidad de las mujeres para decidir sobre sí mismas y su futuro y más cuando han sido expuestas a situaciones de violencia sexual pero sin desconocer que la libertad sexual sin límites es en sí misma una vulneración de la dignidad humana.

Sobre la relación del aborto con la dignidad de la mujer, la Corte Interamericana de Derechos humanos ha precisado en el Caso Atala Riffo y Niñas Vs. Chile. Fondo. Sentencia de 24 de febrero, que (2012):

\section{Exigirle a la madre que condicionara sus opciones de vida implica utilizar una concepción "tradicional" sobre el rol social de las mujeres como madres, según la cual se espera socialmente que las mujeres lleven la responsabilidad principal en la crianza de sus hijos e hijas y que en pos de esto hubiera debido privilegiar la crianza de los niños y niñas renunciando a un aspecto esencial de su identidad.}

$\mathrm{Si}$ tradicionalmente la figura antropocentrista privilegia el rol de la mujer como madre y cuidadora de los hijos, a pesar de reconfigurarse esta visión tradicional y permitir concebir a la mujer en otros roles sociales, es claro que la mujer como dadora de vida si es la primera implicada en la vida que nace de si, es ella la portadora. Lo anterior tiene mayor sentido si consideramos que el tema de la dignidad humana de la mujer en condiciones de equidad no solo compete al libre desarrollo de la personalidad, sino también al trato y el respeto por la vida del otro, el análisis filosófico que podría entreverse en este sentido se evidencia en la
Sentencia C-221 de 1994 de la dosis personal (como se expresa también en la Sentencia C-404 de 1998). En dicha ocasión, Carlos Gaviria fue ponente y en el salvamento de voto dijo sobre Kant y la dignidad humana que:

Emmanuel Kant advierte en torno a la finalidad del ser humano, que el libre albedrío no puede tener su esencia sino en la realización de los fines racionales del hombre. La finalidad de que habla el filósofo alemán es la finalidad de la naturaleza; dicha finalidad no es otra que el mismo hombre, ya que éste es "el único ser sobre la tierra que posee un entendimiento $\mathrm{y}$, por tanto, una facultad de proponerse unos fines, por eso merece ciertamente el título de señor de la naturaleza, y si se considera a la naturaleza como a un sistema teleológico, es según su destino, el fin último de la naturaleza; pero es solamente de una manera condicional, es decir, a condición de que sepa y de que tenga la voluntad de establecer entre ella y él una relación final tal, que ésta sea independiente de la naturaleza y, bastándose a sí misma, pueda ser por consiguiente fin último ( Corte Constitucional, Sentencia C-221 de 1994, p.1)

La libertad humana funciona como condicionamiento y como determinación de la existencia de otras realidades, es por ello que la libertad, la autonomía y la voluntad logran empoderar a la mujer de su realidad. La libertad es el resultado de un imperativo ético necesario y determinante en los seres humanos y, por ello, contribuye a los fines supremos de la mujer, entre ellos, la equidad.

Para Kant, la ley no es un resultado solamente externo al hombre; la ley que dirige a la razón debe permitir conducir el 
pensamiento hacia las tendencias del ser humano hacia sus fines propios y con ello servir como medida de los mismos actos humanos. Es por eso que lo fundamental de la libertad es el deber de conservarla. Pero a la vez, al ser humano concebirlo como un ser de fines, la libertad es un despliegue del ser personal y tal facultad se encauza a los fines del ser humano. Lo anterior indica que, si bien la mujer puede buscar y defender libremente su equidad y autonomía, no por ello puede transgredir la autonomía y la vida de los demás actores de la sociedad. Es válida la lucha por los derechos, pero no bajo la legitimación de la violación de otros derechos como son los de los niños.

La mujer se piensa bioéticamente cuando admite que es un ser que vive en sociedad, cuando busca ser respetada y respeta a los otros, cuando busca defender sus derechos y luchar contra las prácticas de discriminación, cuando reconoce que si bien, ser digna es ser libre y autónoma, su dignidad tiene un límite y es el respeto por la dignidad de los demás seres humanos. Sostiene Perojo Páez (2013) que:

La necesidad de divulgar la bioética ha sido un tema recurrente por la Organización Panamericana de la Salud, particularmente La Agenda de Salud para las Américas (20082017) enfatizó en que tienen que propagarse sus principios en función de proteger el respeto a la dignidad de las personas, salvaguardar la diversidad cultural y la aplicación de los conocimientos en salud. (p.398)

Desde tal perspectiva y alcance de la bioética, el principio de dignidad humana es una defensa de la vida, porque si la muerte es un medio para conseguir un fin de libertad, entonces se haría del ser humano un medio para la libertad, es decir, un actor legitimado para limitar a su voluntad otras libertades.
Desde esta perspectiva, la equidad de la mujer es una lucha por la vida digna, el respeto y la inclusión, pero el aborto sobrepasa el concepto de dignidad humana porque legitima a la libertad para trasgredir sobre la vida, porque busca alcanzar un fin que no es la vida sino la permanencia de la libertad.

Si tenemos en cuenta que el derecho de decisión es una potestad que tiene la mujer sobre sus libertades reproductivas y sobre su cuerpo, entendemos entonces que esta libertad "termina donde comienzan los derechos del nasciturus, cuyo límite se presenta en el instante mismo de la fecundación, el aborto no puede considerarse como un derecho humano" (Jesús Zarate, 2014, p. 21) en tanto vulnera la dignidad del ser humano que está por nacer, pero en los casos en los que el aborto defiende la dignidad humana del nasciturus (malformación del feto) tiene sentido bioético que continúe su aceptación.

El reto que tiene la mujer en la búsqueda de una sociedad equitativa es "aprender a vivir con el otro a medida que crecemos y avanzamos por la vida, como suele decirse, es al parecer igualmente válido para las grandes federaciones de la humanidad, para los pueblos y Estados" (Gadamer, 1990, p.37) y para todos los grupos sociales. Equidad no es solo luchar autónomamente por la propia libertad sino aprender que se vive con otros y que mientras se pisoteen otras dignidades para alcanzar la propia, la equidad seguirá siendo una utopía.

La mujer no tiene una esencia definitiva, por eso debe construirse siempre para incluirse, visibilizarse en sus derechos y los de todos aquellos que no tienen voz. La tarea bioética de la mujer hoy (quien por años ha sido el principal artífice de la lucha por la equidad) es develar los rostros oprimidos, elevarlos y demostrar una renuncia a la eliminación del otro para satisfacción de la propia libertad. Dignidad humana implica libertad, pero ser libre es 
Bioethics and women: A iusphilosophical analysis of human dignity as equity condition.

someterse eternamente a la renuncia de sí mismo y de otros.

\section{CONCLUSIONES}

El principio de dignidad humana es un elemento de análisis y protección esencial a la hora de analizar un derecho como el de la libertad sexual y reproductiva, toda vez que en él convergen la libertad, la protección de la vida y la autonomía. La libre determinación no puede ser un disposición absoluta que supere el sentido mismo del respeto por la vida humana.

La dignificación de la mujer inicia en asumirse como un ser humano con conciencia humana y con amor por lo humano. Respetarse, construirse y asumirse individual y colectivamente debe ser un proceso que no afecte la vida humana y su libertad, la cual es un derecho que se adquiere desde el vientre y que se ve vulnerado por una práctica como el aborto.

La bioética en la mujer implica una educación en el manejo ético de su vida y ello incluye su salud mental y corporal. Esa educación debe permitirle no solo asumirse en libertad y respeto para si misma sino que debe generarle conciencia de la importancia de establecer equitativamente el mismo respeto por la libertad y dignidad de otros seres humanos.

\section{FUENTES DE INFORMACIÓN}

\section{Fuentes bibliográficas}

Casado, M, Luna, F y Vásquez, R. (2013) Género y Bioética. México: Fontamara.

Delgado Díaz, C.(2008). Revolución científica y Bioética. Cuba: Félix Varela.

Gadamer, H (1990) La herencia de Europa. España: Ed. Península.

Kant, I. (2007) Fundamentación de la metafísica de las costumbres. Puerto Rico: Ed. Pedro M. Rosario.
Londoño Berrío, H (2012) Constitución y Derecho penal de las minorías étnicas. Conferencia, pronunciada en el IV Congreso Internacional de Derecho Penal. Universidad Sergio Arboleda. Seccional Santa Marta. Santa Marta, 5 de octubre de 2012

Rilke, J. (1956) Poemas a la noche y otra poesía póstuma y dispersa. Espa ebook.

\section{Fuentes hemerográficas}

Boscán Leal, A. (2015). La Bioética Feminista y la construcción actual del género. En: Opción. (Vol. 31, núm. 5),. 162-188.

Figueroa Yáñez, G. (2017). Bioética de género en medicina: Fundamentos $\mathrm{y}$ desafíos. En: Acta Bioethica 23 (1): 83-90.

Fuller. (2008). La perspectiva de género y la criminología: una relación prolífica. Revista Tábula Rasa. (8) 97-110.

Gil Millán , M.(2015) La violencia sexual como un atentado contra la dignidad de la mujer. En: Revista de derecho UNED. (núm. 17)

Gómez Lamont, M.F. (2016). Bioética, feminismo y la dignidad de las "mujeres": proceso social y cambio de valores en las terapias familiares. En: Alternativas Psicológicas.(N.36). Universidad Nacional Autónoma de México.

Miller, A. M. y Roseman, M. J. (2011). Sexual and reproductive rights at the United Nations: frustration or fulfilment? Reproductive Health Matters, 19(38), 102118.

\section{Fuentes electrónicas}

Convención Interamericana para Prevenir, sancionar y erradicar la violencia contra la mujer "Convención de Belem do Pará"(1994). Recuperado de : 
http://www.oas.org/juridico/spanish/tratad os/a-61.html

Jesús Zárate Cuello, A. (2014). ¿Es el aborto un derecho sexual y reproductivo de la mujer? Análisis desde el bioderecho, la Bioética, la biopolítica y la biojurídica en Estados Unidos, España y Colombia. Revista Latinoamericana de Bioética, 14(2). Recuperado de : http://search.ebscohost.com.consultaremot a.upb.edu.co/login.asp $\mathrm{x}$ ?direct $=$ true $\& \mathrm{db}=\mathrm{f}$ ua\&AN=108474152\&lang $=$ es $\&$ site $=$ edslive

Perojo Páez, V. M. (2015). La violencia contra la mujer: abordaje en profesionales de la Atención Primaria desde una perspectiva bioética. Revista Cubana de Medicina General Integral, 31(4). Recuperado de: http://search.ebscohost.com/login.aspx?dir ect $=$ true $\& d b=a 9 h \& A N=116562951 \&$ lang $=$ es \&site $=$ eds-live

\section{Jurisprudencia}

Corte IDH. (2012). Caso Atala Riffo y Niñas Vs. Chile. Fondo, Reparaciones y Costas. Sentencia de 24 de febrero de 2012.

Corte IDH. (2016) Caso I.V. Vs. Bolivia. Excepciones Preliminares, Fondo, Reparaciones y Costas. Sentencia de 30 de noviembre de 2016. Serie C No. 329.

Corte Constitucional, Sala séptima de Revisión (1992). Sentencia T-222 de 1992. (M.P. Ciro Angarita Baron)

Corte Constitucional, Sala Plena (28 de Octubre de 1993). Sentencia T-493 de 1993. (M.P. Antonio Barrera Carbonell)

Corte Constitucional, Sala Plena (5 de mayo 1994). Sentencia C-221 de 1994. (M.P. Carlos Gaviria Díaz)

Corte Constitucional, Sala Plena (10 de Agosto de 1998). Sentencia C-404 de 1998.
(M.P Carlos Gaviria Díaz y Eduardo Cifuentes Muñoz). 